\title{
Multiple Reference Frames for Motion in the Primate Cerebellum
}

\author{
Aasef G. Shaikh, Hui Meng, and Dora E. Angelaki \\ Department of Neurobiology, Washington University School of Medicine, St. Louis, Missouri 63110
}

\begin{abstract}
Knowledge of body motion through space is necessary for spatial orientation, self-motion perception, and postural control. Yet, sensory afferent signals may not directly provide such information to the brain. Because motion detected by the vestibular end organs is encoded in a head-fixed frame of reference, a coordinate transformation is thus required to encode body motion. In this study, we investigated whether cerebellar motion-sensitive neurons encode the translation of the body through space. We systematically changed both the direction of motion relative to the body and the static orientation of the head relative to the trunk. The activities of motion-sensitive neurons in the most medial of the deep cerebellar nuclei, the rostral fastigial nucleus, were compared with those in the brainstem vestibular nuclei. We found a distributed representation of reference frames for motion in the rostral fastigial nucleus, in contrast to cells in the vestibular nuclei, which primarily encoded motion in a head-fixed reference frame. This differential representation of motionrelated information implies potential differences in the functional roles of these areas.
\end{abstract}

Key words: self-motion; cerebellum; frame of reference; sensorimotor; multisensory; gain field; coordinate transformation

\section{Introduction}

The vestibular system forms an essential component in the sensing and generation of movement. Our inertial motion sensors, the otolith organs, located in the inner ear cavity, are highly conserved throughout evolution and function to provide an accurate moment-to-moment estimate of our motion through space. Being fixed in the head, the corresponding signals carried to the brain by primary vestibular afferents detect self-motion in a head-fixed coordinate system. However, a reference frame transformation might take place centrally because day-to-day functions often require knowledge of body position, orientation, and movement. For example, systematic alterations in vestibulospinal reflex properties after altered static orientations of headon-trunk have been reported (Nashner and Wolfson, 1974; Kennedy and Inglis, 2002). In addition, information on body orientation and movement is also important for the perception of self-motion and location of objects in extra-personal space (Lackner and Graybiel, 1978; Mergner et al., 1991, 1992).

How does the brain acquire access to an internal estimate of body motion if this information is not provided by primary vestibular afferents? By combining otolith signals, which encode translation in head coordinates, with neck proprioceptive information that signals the position of the head relative to the body, a coordinate transformation could take place, whereby the brain constructs an internal estimate of body motion through space.

\footnotetext{
Received Jan. 11, 2004; revised March 8, 2004; accepted March 30, 2004.

This work was supported by grants from the National Institutes of Health (DC04260, F32 DC006540) and NASA (NAG2-1493).

Correspondence should be addressed to Dr. Dora Angelaki, Department of Anatomy and Neurobiology, P.O. Box 8108, Washington University School of Medicine, 660 South Euclid Avenue, St. Louis, M0 63110. E-mail: angelaki@pcg.wustl.edu.

D0I:10.1523/JNEUROSCI.0109-04.2004

Copyright $\odot 2004$ Society for Neuroscience $\quad$ 0270-6474/04/244491-07\$15.00/0
}

Extensive convergence of vestibular and somatosensory signals has been reported in motion-sensitive areas of the brainstem vestibular nuclei (VN), cerebellar cortex (e.g., anterior and posterior cerebellar vermis), and deep cerebellar nuclei (Boyle and Pompeiano, 1981; Anastasopoulos and Mergner, 1982; Wilson et al., 1990; Manzoni et al., 1998, 1999; Gdowski and McCrea, 1999, 2000; McCrea et al., 1999). At present, it is not known whether this convergence reflects an underlying reference frame transformation to compute body motion in space. Here, we specifically address one of the possible functions of these multisensory interactions. Results provide novel evidence that a population of neurons in the most medial of the deep cerebellar nuclei, the rostral fastigial nucleus (FN), encode the translation of the body through space. In contrast, motion-sensitive cells in the VN seem to maintain a head-fixed reference frame for encoding self-motion.

\section{Materials and Methods}

Single-unit activities in the rostral FN and rostral medial and lateral VN were recorded in two juvenile monkeys (Maccaca Fascicularis), which were chronically implanted with an eye coil and a circular delrin ring for head stabilization. A guide tube delrin platform with a staggered array of holes (Angelaki and Dickman, 2000; Dickman and Angelaki, 2002) was stereotaxically secured to the skull inside the head ring. To provide better access to the medial cerebellar nuclei, the platform was slanted in the horizontal plane $10^{\circ}$ from anterior to posterior and $10^{\circ}$ from left to right. All surgical procedures were performed under sterile conditions in accordance with institutional and National Institutes of Health guidelines.

During experiments, the monkeys were seated in a primate chair with their bodies secured with multiple shoulder and lap belts while their extremities were tied to the chair. Their head ring was fixed to the primate chair through a 2.5 inch center bore-bearing piece that could be locked in place at different head-on-trunk orientations. The primate chair was then secured inside the inner frame of a vestibular turntable consisting of a three-dimensional rotator on top of a $2 \mathrm{~m}$ linear sled (Acutronics, Pittsburgh, PA). The linear acceleration was measured with a three-axis 
accelerometer mounted on the primate chair. Thus, as their trunk and extremities were softly restrained to the chair, the motion measured by the accelerometer reflected the motion of the body through space. We point out that in this presentation, body refers to the single block of torso that always faced forward relative to the primate chair.

For each recording session, the eye coil signals and the linear accelerometer output were low-pass filtered $(200 \mathrm{~Hz}$; 6-pole bessel), digitized at a rate of $833.33 \mathrm{~Hz}$ (model 1401, 16-bit resolution; Cambridge Electronics Design, Cambridge, UK), and stored for off-line analysis. Extracellular recordings from single $\mathrm{FN}$ and $\mathrm{VN}$ neurons were obtained with epoxy-coated tungsten microelectrodes inserted into 26-gauge guide tubes. Neural activity was amplified, filtered $(300 \mathrm{~Hz}$ to $6 \mathrm{kHz}$ ), and passed through a BAK Instruments (Germantown, MD) dual-time amplitude window discriminator. Single-unit spikes triggered acceptance pulses (BAK window discriminator) that were stored on a computer using the event channel of a Cambridge Electronics Design (model 1401) data acquisition system. In addition, eye movement signals, stimuli, and neural activity were stored on a digital audio tape recorder for off-line spike discrimination. Stimulus protocols and data acquisition were computer controlled using scripts written for the Spike2 (Cambridge Electronics Design) software environment.

During initial experiments in each animal, the abducens nuclei were first identified bilaterally. The recording sites in the FN and VN were then identified on the basis of their stereotaxic location relative to the abducens nuclei and the fourth ventricle. All neural responses were obtained from vestibular-only $(\mathrm{VO})$ neurons. These neurons were recorded from rostral portions of the medial and lateral $\mathrm{VN}$ within $1 \mathrm{~mm}$ of areas where eye movement-sensitive cells were recorded (Angelaki et al., 2001; Dickman and Angelaki, 2002). In the FN, neurons were recorded in the rostral portions of the nucleus, anterior to all pursuit-related and saccaderelated cells that we consistently encountered in the caudal FN (Gardner and Fuchs, 1975; Buttner et al., 1991). To characterize cells as VO or eye movement-sensitive, a standard protocol was used, including rotations, fixations, and smooth pursuit eye movements (Angelaki et al., 2001).

$\mathrm{VN}$ and FN cells that were sensitive to linear motion also were tested using different directions of translation $\left(0,30,60,90,120,150\right.$, and $180^{\circ}$; defined relative to the body) (see Fig. 1), with the head secured at three different positions relative to the trunk (see Fig. 1, monkey schematic drawing). Initially, the animal's head was straight-ahead relative to the body $\left(h=0^{\circ}\right)$ (see Fig. 1, blue drawing). Subsequently, using the specially constructed head holder, the head was secured $30^{\circ}$ to the left relative to the animal's body $\left(h=30^{\circ}\right)$ (see Fig. 1, green drawing), and the sixdirection translation protocol was repeated. Finally, the motion protocol was also delivered with the animal's head secured $30^{\circ}$ to the right $(h=$ $-30^{\circ}$ ) (see Fig. 1, red drawing). The order in which these three head-ontrunk positions were tested was pseudo-randomized for different neurons. Such reorientations of the head in relation to the body were intended to dissociate between body and head coordinates. Data were collected during sinusoidal translational motion stimulation either at 0.5 or $2 \mathrm{~Hz}\left( \pm 0.2 \mathrm{~g}\right.$, with $\left.g=9.81 \mathrm{~m} / \mathrm{sec}^{2}\right)$, whichever frequency provided the largest cell modulation. Results were similar at the two frequencies and thus are presented together.

Data analyses were performed off-line using custom-written scripts in Matlab (MathWorks, Natick, MA). For each action potential, an occurrence time was logged. Instantaneous frequency (IFR) was then calculated as $1 /$ (interspike interval) and assigned to the middle of the interval. For each stimulus cycle (e.g., $n$th cycle), an integral $(n-1)$ times the period was subtracted from the timing for all of the instantaneous frequency values for that specific cycle. The result was to "fold" all instantaneous frequency values into a single stimulus cycle. This procedure provides no averaging, because all spike occurrences are represented in time. Neural response amplitude and phase were then determined by fitting a sine function to both response and stimulus using a nonlinear least squares (Levenberg-Marquardt) minimization algorithm. Portions of the response cycle in which no spikes occur (silencing or rectification) does not pose a problem to the fitting procedure, because only positive IFR values exist in the response cycle and are weighed in the least-squares estimations. Examples of such fits have been illustrated for two cells in Figure 1. Neural response gains were expressed as spikes/second/gravity.
Phase was the difference (in degrees) between peak neural activity and peak linear acceleration.

Neural response gains and phases were subsequently plotted as a function of the direction of translation and fitted (Levenberg-Marquardt optimization method) with a two-dimensional spatiotemporal model that represents a more generalized model than cosine tuning (Angelaki and Dickman, 2000). This fitting procedure allowed the estimation of four parameters: direction, gain, and phase of the maximum response as well as the minimum response gain (Angelaki, 1991; Angelaki and Dickman, 2000). The goodness of fit was based on the variance-accounted-for $(\mathrm{VAF})$, computed as VAF $=\{1-[\operatorname{var}($ model - data $) / \operatorname{var}($ data $)]\}$. The fitting procedure was first applied to each head-on-trunk position separately (analysis step I). This allowed estimation of all four response parameters, separately for each of the three head-on-trunk positions. This first step in analysis was necessary to investigate which, if any, spatial tuning properties changed as a function of head-on-body position. Once it was established that the only systematic difference was a spatial shift of the tuning curves, data in all three head-on-trunk positions were also fitted simultaneously by assuming that the maximum response orientation for each cell was shifted through an angle, $\pm \lambda 30^{\circ}$, for the rotated head-in-trunk positions (analysis steps II and III). Thus, in a second stage of analysis (step II), all data for each cell were fitted simultaneously with each of two four-parameter models corresponding to the body-fixed reference frame model $(\lambda=0$; i.e., no spatial shift of maximum response direction for different head-on-trunk positions) or the head-fixed reference frame model $\left(\lambda=1\right.$; i.e., $\mathrm{a} \pm 30^{\circ}$ systematic shift in the maximum response direction for different head-on-trunk positions). Finally, because it is possible that cells exhibit an in-between behavior, a third fitting procedure was used, where $\lambda$ was allowed to vary and was one of the parameters fitted (analysis step III). This latter $\lambda$-variable model had a total of five parameters. A sequential $F$ test (Draper and Smith, 1998) was used to ascertain the statistical significance of increasing the model parameters by comparing the errors associated with the $\lambda$ variable and each of the two $\lambda$-fixed models while allowing for differences in their degrees of freedom. The sequential $F$ test was the statistical test used to quantify, on a cell-by-cell basis, whether the tuning of each neuron was best described by the head-fixed/body-fixed or an intermediate reference frame model. For both the $\lambda$-fixed and $\lambda$-variable models (analysis steps II and III), all other parameters of cell tuning (e.g., maximum and minimum response gain and phase) were independent of head-on-trunk position.

\section{Results}

The head and body coordinate systems were dissociated by systematically varying both the direction of motion and the static orientation of the head relative to the body (Fig. 1). Neural activities were recorded as animals were translated along different directions $\left(0,30,60,90,120,150\right.$, and $180^{\circ}$; defined relative to their body) in the horizontal plane with the head fixed to the trunk in one of three different positions: straight-ahead (Fig. 1, blue traces) $\left(h=0^{\circ}\right)$ as well as rotated $30^{\circ}$ to the left $\left(h=30^{\circ}\right)$ or right $\left(h=-30^{\circ}\right)$ (Fig. 1, green and red traces, respectively). Accordingly, if a neuron encodes the motion of the body, its spatial tuning should be independent of the change in head position. In contrast, if a cell exclusively detects the motion of the head independently of body motion, its preferred movement direction should systematically shift to the left or right to reflect the shifted direction of motion in head coordinates (Fig. 1, monkey head orientation drawing). Neurons with properties reflecting either the body or the head coordinate systems were encountered in the rostral fastigial nucleus, as illustrated for the two cells in Figure $1 A$ (body coordinates) and Figure $1 B$ (head coordinates). For the neuron illustrated in Figure $1 A$, the directions of maximum and minimum neural response modulation $\left(0\right.$ and $90^{\circ}$ motion directions, respectively) were the same for all three head-on-body positions. In contrast, for the neuron in Figure $1 B$, the directions of maximum and minimum response modulation shifted for the 


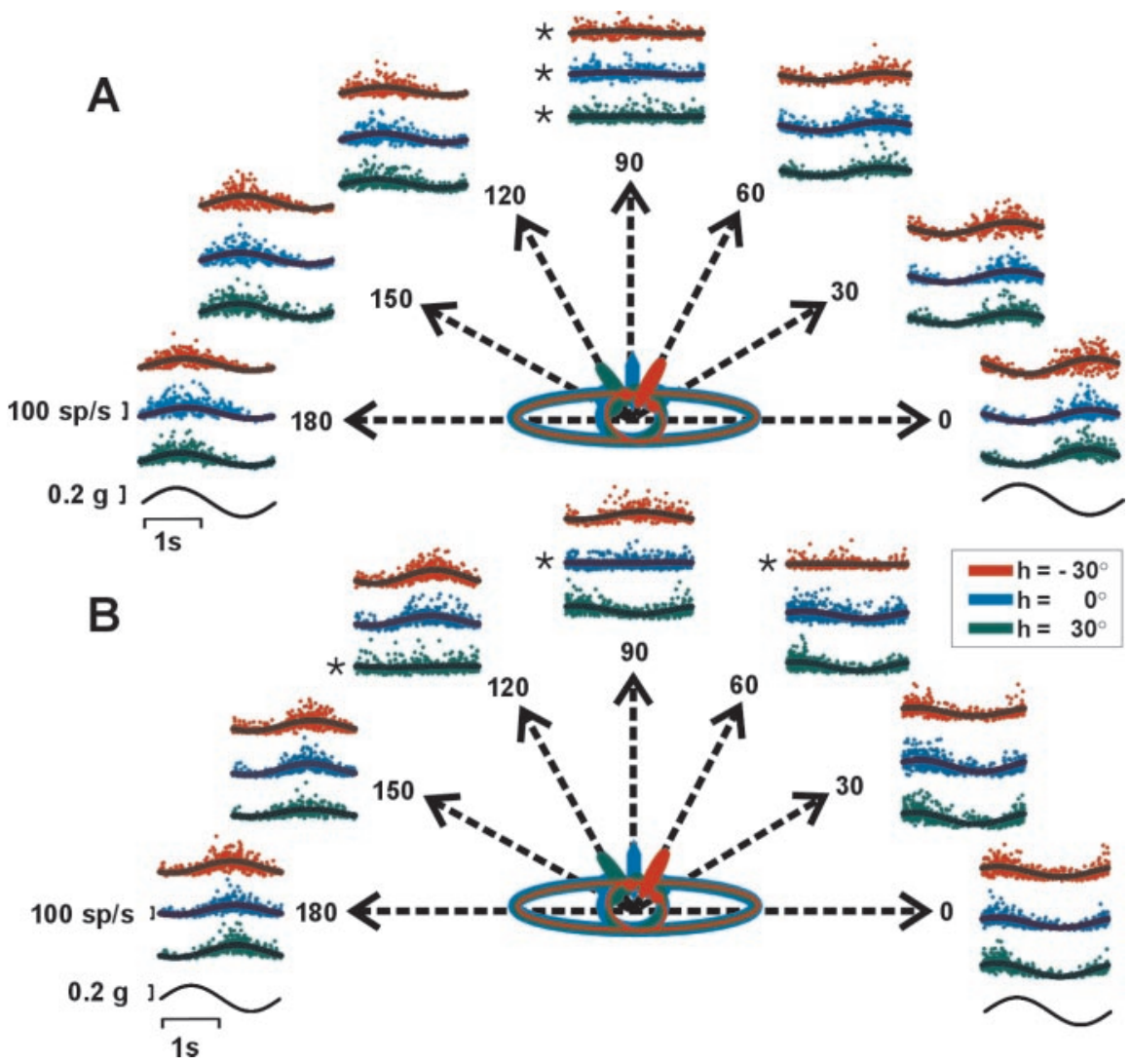

Figure 1. Instantaneous firing rates of two rostral FN neurons during passive whole-body translation at $0.5 \mathrm{~Hz}( \pm 0.2 \mathrm{~g})$ along different directions in the horizontal plane. Data are color coded for the three head-on-body positions; blue, red, and green colors are used for straight-ahead $\left(h=0^{\circ}\right)$ and $30^{\circ}$ to the right $\left(h=-30^{\circ}\right)$ or left $\left(h=30^{\circ}\right)$ head-on-trunk positions. Superimposed solid lines represent best-fit sine functions. $A$, FN neuron encoding motion in a body reference frame, where the firing rate of the cell is independent of head-on-trunk position. $B$, FN neuron encoding motion in a head reference frame. The firing rate of the cell changes for the different head-on-trunk positions. In both panels, the minimum responses are marked with asterisks. The black traces represent the linear acceleration stimulus. Motion stimuli are defined relative to the body and, thus, change direction relative to the head (monkey drawing).

three different head-on-body positions, such that they would remain fixed relative to the head. This shift is better seen for the minimum response direction, which shifts from $90^{\circ}\left(h=0^{\circ}\right)$ (Fig. 1 , blue traces) to $120^{\circ}\left(h=30^{\circ}\right)$ (Fig. 1, green traces) and $60^{\circ}(h=$ $-30^{\circ}$ ) (Fig. 1, red traces), shown with asterisks in Figure $1 B$.

To describe these differences, neural response gains and phases were plotted as a function of motion direction separately for each of the three head-on-body positions (Fig. 2). The spatial tuning curves for the three head-on-body positions superimpose for the neuron shown in Figure $2 A$, suggesting that this cell encodes the translational motion of the body (i.e., encoding linear acceleration in body coordinates). In contrast, the spatial tuning for the neuron in Figure $2 B$ shifts for the three head-on-body positions, indicating that this cell encodes the translational motion of the head. To quantify these observations, a spatiotemporal tuning model was used to fit neural gain and phase as a function of motion direction (Angelaki, 1991; Angelaki and Dickman, 2000). Three different analysis steps were performed. First, the spatiotemporal model was fitted separately to data for each headon-trunk position (analysis step I). This allowed an independent estimation of the gain and phase of neural firing in response to motion along the maximum response direction of the neuron for each head-on-body position. Figure $3 A$ illustrates these values for each of the two rotated head-on-body positions $\left(h= \pm 30^{\circ}\right)$ plotted versus the corresponding values when the head was straight- ahead relative to the body $\left(h=0^{\circ}\right)$. There was no systematic dependence of any of these parameters on static neck inputs, as illustrated by linear regression (Fig. 3A, solid lines; dashed lines illustrate $95 \%$ confidence intervals). The main effect of changing the head-on-body position was a systematic shift in the spatial tuning curves, including the maximum and minimum response directions. Figure $3 B$ illustrates the mean spatial tuning curve shift, plotted separately for the cerebellar and brainstem populations (gray squares vs open circles, respectively). Only the VN data were consistent with the spatial shift expected from a head-fixed reference frame coding of motion. The difference in the spatial shift of the tuning curves for FN and VN neurons was statistically significant $\left(F_{(1,31)}=11.4 ; p<0.01\right)$.

Because other parameters of cell firing rate properties remained independent of changes in the head-on-trunk position (including the maximum gain of the cells), the spatiotemporal model was next used to fit the neural gain and phase dependence on motion direction for all three head-ontrunk positions simultaneously. According to the population behaviors in Figure $3 B$, the maximum sensitivity direction of each cell was assumed to shift through an angle $\pm \lambda 30^{\circ}$ for the rotated head-ontrunk positions. Thus, in a second analysis procedure (step II; see Materials and Methods), the value of $\lambda$ was fixed to either $\lambda=0$ (body-fixed reference frame model; e.g., no changes in the direction of maximum response gain as a function of head-on trunk orientation) or $\lambda=1$ (head-fixed reference frame model; i.e., allowing for a $\pm 30^{\circ}$ shift in the maximum response direction as a function of head-on-trunk position). The VAF values for each of the head-fixed and body-fixed reference frame models were compared for each cell in Figure $4 A$. In the figure, each symbol corresponds to one cell in either the VN (open circles) or the FN (gray squares), and the dotted line illustrates the unity-slope line. For all but one of the VN neurons, the headfixed reference frame model gave as good or higher VAF values than the corresponding body-fixed reference frame model (Fig. $4 A$; all but one of the open circles fall in the top left half of the diagram). In contrast, only approximately one-third of the FN neurons was better fitted with the head-fixed reference frame model. The majority of the FN cells better complied with the body-fixed reference frame model. However, many neurons were equally well fitted by either model, illustrating the need for the third analysis procedure.

Thus, to further investigate the reference frame used by FN and $\mathrm{VN}$ neurons to encode motion, an additional analysis was performed where the value of $\lambda$ was allowed to vary and was actually one of the parameters fitted for data of each cell (analysis step III; see Materials and Methods). This resulted in a model with one additional parameter. To quantify whether this higher parameter model fitted the data better compared with the lower parameter head-fixed or body-fixed model (step II), a sequential 


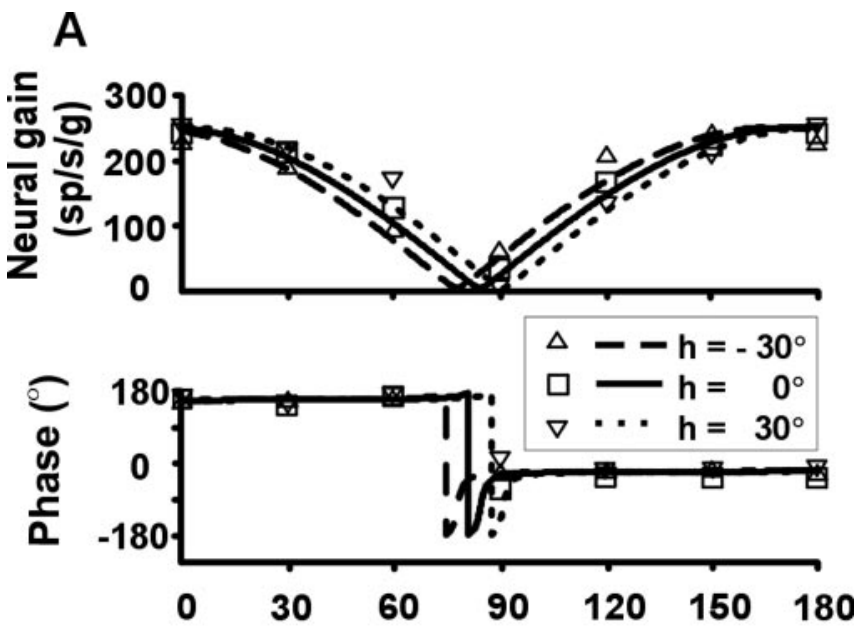

B
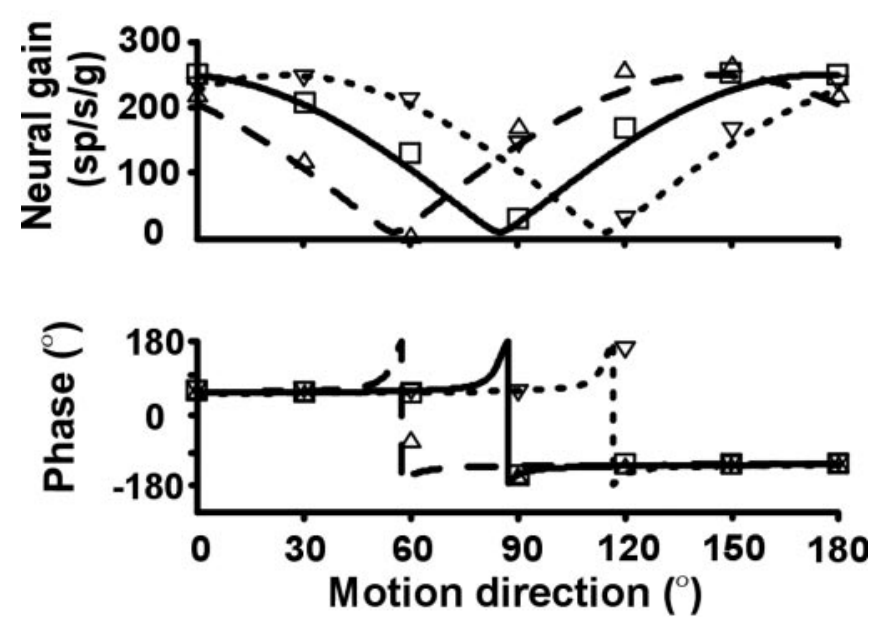

Figure 2. Neural response gain and phase plotted as a function of the direction of translation for each of the three head-on-body positions. A, FN neuron encoding motion in a body reference frame (same data as in Fig. 1A). The firing rate of the cell is independent of head-on-trunk position. $B$, FN neuron encoding motion in a head reference frame (same data as in Fig. $1 B$ ). The firing rate of the cell changes for the different head-on-trunk positions. Different symbols are used for data obtained for the three head-on-trunk positions. The corresponding lines illustrate the fit of the $\lambda$-variable spatiotemporal tuning model. sp/sec/g, Spikes/second/gravity.

$F$ test was used (Draper and Smith, 1998). The fitted $\lambda$ values are summarized in Figure $4 B$. For neurons encoding motion in a body reference frame, the spatial shift parameter, $\lambda$, was close to zero. This was the case for many FN but not VN neurons. In $43 \%$ (15 of 35 ) of the FN cells, but only one VN cell, the $\lambda$-variable model fit was better $(p<0.05)$ than the head-fixed model fit $(\lambda=1)$ and statistically indistinguishable $(p>0.05)$ from the body-fixed model fit $(\lambda=0)$. Therefore, this subpopulation of FN cells encodes motion in a body-fixed reference frame. The opposite result, consistent with the head-fixed reference frame predictions, was encountered in 34\% (12 of 35) of the FN cells and $80 \%$ (14 of 20 ) of the VN cells. Thus, the majority of VN neurons and a subpopulation of FN cells encode motion in a head-fixed reference frame. The remaining neurons (eight FN and five VN cells) did not favor either one of the $\lambda$-fixed models. These neurons had $0.4<\lambda<0.7$ and complied with neither the head-fixed nor the body-fixed coordinate system predictions, suggesting an intermediate reference frame. The difference in the distributions for the spatial shift parameter, $\lambda$, between the FN and $\mathrm{VN}$ cell populations was statistically significant $\left(\chi^{2}=23.5\right.$; $\mathrm{df}=14 ; p=0.05)$, having a median value of 0.4 and 0.9 , respectively.

\section{Discussion}

These results demonstrate for the first time that subcortical motion-sensitive neurons exist that encode translation of the body, unlike primary vestibular afferents, which detect motion in a head-fixed reference frame (Cullen and Minor, 2002). Interestingly, the body reference frame was primarily observed in the deep cerebellar nuclei, where approximately half of the FN cells encoded motion of the body through space. A similar result was not seen in brainstem neurons, raising the possibility that the coordinate transformation might take place in the cerebellar cortex. The rostral fastigial nucleus represents the main output of the medial zone of the anterior vermis (Voogd, 1989), the role of which in vestibular-somatosensory interactions has received strong experimental support (Manzoni et al., 1998, 1999). In fact, Manzoni et al. (1999) have previously reported that static neck input modulates the responses of anterior vermis Purkinje cells during complex vestibular stimulation in decerebrate cats. The present results show that these vestibular-somatosensory interactions implement a coordinate transformation to estimate motion of the body through space. The outcome of the reference frame change is observed in the firing rates of a subpopulation of FN neurons, an area that represents a main cerebellar outflow to the spinal cord, premotor brainstem centers, and the thalamocortical system.

\section{Vestibular-somatosensory convergence}

The concept of vestibular-proprioceptive interactions was originally introduced by von Holst and Mittelstaedt (1950). Several lines of evidence have supported this idea. First and foremost, control of muscular coordination in relationship to movement is crucial for maintaining posture. Vestibular-proprioceptive interactions have been reported for limb control (Manzoni et al., 1983; Ezure and Wilson, 1984; Wilson et al., 1986) and neck reflexes (Peterson et al., 1985). In addition, sensations of tilting and falling, as well as altered perception of spatial orientation can be induced by electrical stimulation or vibration of the neck (Karnath et al., 1994; Ivanenko et al., 1999). Additional psychophysical experiments using trunk rotation relative to the head have demonstrated that normal human subjects derive trunk motion perception from a combination of vestibular and neck cues (Mergner et al., 1983, 1991). In addition, vibration of muscles or muscle tendons causes proprioceptive "misinformation" producing an illusory sensation of movement (Goodwin et al., 1972). In normal subjects, vibration applied to the neck muscles causes a displacement of body orientation, a shift of subjective midline, and a consistent error in pointing and body sway (Biguer et al., 1988; Pyykko et al., 1989; Karnath et al., 1994).

The interaction between vestibular and joint afferents already occurs at the level of the vestibular nuclei, the first central synapse of primary vestibular afferents (Boyle and Pompeiano, 1981; Anastasopoulos and Mergner, 1982; Wilson et al., 1990). Many VN neurons respond to both vestibular and proprioceptive stimulation (Brink et al., 1980; Kasper et al., 1988). Given such extensive vestibular-proprioceptive convergence in the VN and the fact that the paramedian zone of the anterior vermis projects directly to the lateral vestibular nucleus (Voogd, 1989), the observation that the VN cell population described here used a motion reference frame that was not body-fixed might appear surprising. Several studies have demonstrated recently that there are differences in the $\mathrm{VN}$ neuron responses during rotations in which 

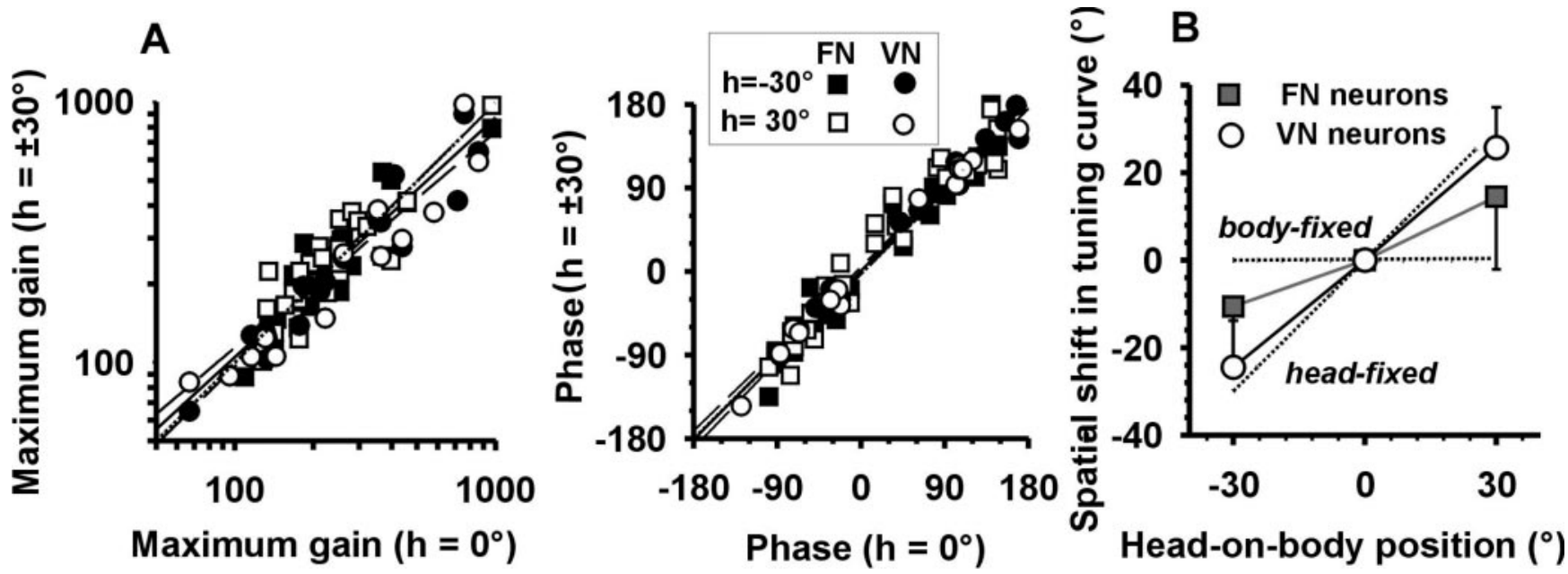

Figure 3. Parameters of a spatiotemporal tuning model fitted to gain and phase as a function of motion direction separately for each head-on-trunk position (analysis step I). $A$, The gain and phase of the cell during motion along the maximum response direction for each of the two rotated head-on-body positions $\left(h= \pm 30^{\circ}\right)$ plotted versus the corresponding values when the head was straight ahead relative to the body $\left(h=0^{\circ}\right)$. Each pair of open and filled symbols corresponding to the same abscissa illustrates data from a cell for which the spatial tuning was tested in both left and right head-on-trunk positions. A few cells were only tested either with the left $\left(h=30^{\circ}\right)$ or the right head-on-trunk $\left(h=-30^{\circ}\right)$ positions. Regression equations: $y=0.2+0.92 x, r^{2}=0.85$ (gain); $y=0.7+1.01 x, r^{2}=0.96$ (phase). $B$, The average spatial shift in the tuning curves for $F N$ and VN neurons was plotted as a function of head-on-body position. Data were normalized before the calculation of the averages by subtracting from the spatial angle of the maximum response direction for $h= \pm 30^{\circ}$ the respective angle with the head centered $\left(h=0^{\circ}\right)$.

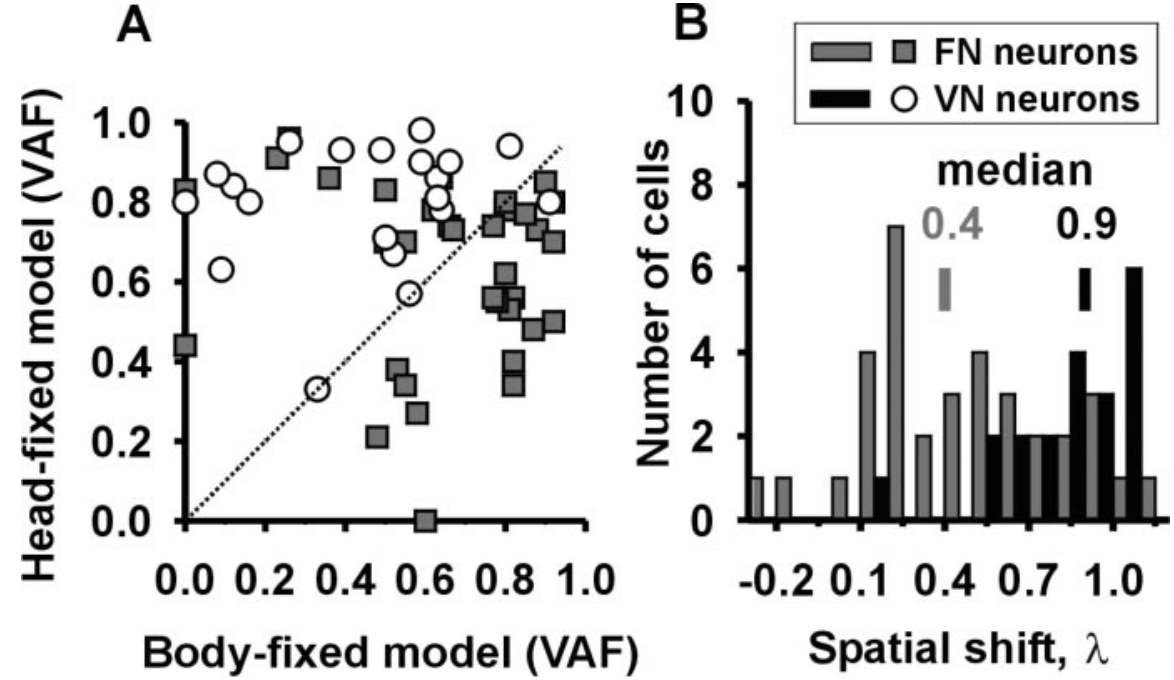

Figure 4. Coordinate frames for FN and VN neurons. $A$, Comparison between the goodness-of-fit (VAF) for two $\lambda$-fixed spatial shift models (analysis step II): a body-fixed reference frame model with $\lambda=0$ (abscissa) and a head-fixed reference frame model with $\lambda=1$ (ordinate). Open circles, VN neurons; gray squares, FN neurons. The dotted line illustrates the unity-slope line. $B_{t}$ Variable spatial shift model (analysis step III): distributions of $\lambda$ values for the VN and FN cell populations (black and gray, respectively). Vertical gray and black lines illustrate the medians of the two populations, $\lambda$ (median for FN neurons) $=0.4$ and $\lambda$ (median for VN neurons) $=0.9$.

the head was fixed relative to the body versus rotations where the head was allowed to rotate freely (Gdowski and McCrea, 1999; McCrea et al., 1999; Roy and Cullen, 2001). Yet, the majority of $\mathrm{VN}$ neurons were closer to head than body coordinates. It is possible that if a clear body reference frame exists in the VN for rotational or translational movements, the location of such neurons might be in more posterior areas than those explored here.

Role of fastigial neurons in motion detection The present experiments, reporting a specific and systematic spatial shift in the tuning curves (without gain or phase changes), represent the first evidence regarding the existence of a body ref- erence frame for translational motion in a subpopulation of FN neurons. Extensive vestibular-proprioceptive interactions that might underlie reference frame transformations also have been reported in rostral fastigial neurons during rotational motion (Buttner et al., 2003). The rostral fastigial nucleus, among all deep cerebellar nuclei, is the one most important in the central processing of motion-related vestibular information. Anatomical and physiological studies have separated the fastigial nucleus into rostral and caudal subdivisions (Noda et al., 1990; Buttner et al., 1991). Neurons of the caudal fastigial nucleus are modulated during saccadic or smooth pursuit eye movements (Gardner and Fuchs, 1975; Buttner et al., 1991; Fuchs et al., 1993). In contrast, the rostral division is populated by neurons that are modulated by vestibular stimulation but do not show sensitivity to eye movements (Gardner and Fuchs, 1975; Buttner et al., 1991; Siebold et al., 1997). Previous studies established that rostral FN neurons respond to sinusoidal rotation in horizontal and vertical planes (Gardner and Fuchs, 1975; Siebold et al., 1997, 1999) as well as to translational movements (Zhou et al., 2001). Given the afferent and efferent projections of rostral FN neurons, it is believed that they are involved in vestibulospinal control, including regulation of gait and postural mechanisms (Gardner and Fuchs, 1975; Buttner et al., 1991; Siebold et al., 1997).

Knowledge of the orientation and motion of the body in space is important for the control of body, limb, and neck posture as well as motion perception and spatial localization of objects in extra-personal space (Mergner et al., 1991, 1992; Horak et al., 1994). All of these functions could be served by projections of FN neurons. For example, the main fastigial efferent projections are 
to spinal-related brainstem structures as well as direct projections to the spinal cord, where they directly synapse on spinal motoneurons (Batton et al., 1977; Fukushima et al., 1977; Wilson et al., 1977). The activities of limb musculature for postural responses during movement have been shown to correlate with body, but not head, displacement as required for postural stability, suggesting that vestibulospinal reflexes might be organized in body coordinates (Manzoni et al., 1998). The same study also showed that intact activity in the anterior vermis is necessary for this function. The spatially shifted reference frames characterizing the tuning of FN neurons might represent a neural substrate for this processing. In addition to spinal projections, the FN nucleus, similar to all other cerebellar nuclei, projects to the thalamus (Batton et al., 1977; Nakano et al., 1980; Asanuma et al., 1983). Although the cortical targets of the FN remain unknown, some studies have suggested FN projections to parietal cortex (Sasaki et al., 1976; Amino et al., 2001) as well as to frontal cortex regions and multimodal visual areas (Kyuhou and Kawaguchi, 1987). Fastigial-thalamocortical projections could thus mediate spatial perception functions in which knowledge of body motion in space is essential.

\section{References}

Amino Y, Kyuhou S, Matsuzaki R, Gemba H (2001) Cerebello-thalamocortical projections to the posterior parietal cortex in the macaque monkey. Neurosci Lett 309:29-32.

Anastasopoulos D, Mergner T (1982) Canal-neck interaction in vestibular nuclear neurons of the cat. Exp Brain Res 46:269-280.

Angelaki DE (1991) Dynamic polarization vector of spatially tuned neurons. IEEE Trans Biomed Eng 38:1053-1060.

Angelaki DE, Dickman JD (2000) Spatiotemporal processing of linear acceleration: primary afferent and central vestibular neuron responses. J Neurophysiol 84:2113-2132.

Angelaki DE, Green AM, Dickman JD (2001) Differential sensorimotor processing of vestibulo-ocular signals during rotation and translation. J Neurosci 21:3968-3985.

Asanuma C, Thach WT, Jones EG (1983) Distribution of cerebellar terminations and their relation to other afferent terminations in the ventral lateral thalamic region of the monkey. Brain Res 286:237-265.

Batton III RR, Jayaraman A, Ruggiero D, Carpenter MB (1977) Fastigial efferent projections in the monkey: an autoradiographic study. J Comp Neurol 174:281-305.

Biguer B, Donaldson IM, Hein A, Jeannerod M (1988) Neck muscle vibration modifies the representation of visual motion and direction in man. Brain 111:1405-1424.

Boyle R, Pompeiano O (1981) Responses of vestibulospinal neurons to neck and macular vestibular inputs in the presence or absence of the paleocerebellum. Ann NY Acad Sci 374:373-394.

Brink EE, Hirai N, Wilson VJ (1980) Influence of neck afferents on vestibulospinal neurons. Exp Brain Res 38:285-292.

Buttner U, Fuchs AF, Markert-Schwab G, Buckmaster P (1991) Fastigial nucleus activity in the alert monkey during slow eye and head movements. J Neurophysiol 65:1360-1371.

Buttner U, Glasauer S, Glonti L, Guan Y, Kipiani E, Kleine J, Siebold C, Tchelidze T, Wilden A (2003) Multimodal signal integration in vestibular neurons of the primate fastigial nucleus. Ann NY Acad Sci 1004:241-251.

Cullen KE, Minor LB (2002) Semicircular canal afferents similarly encode active and passive head-on-body rotations: implications for the role of vestibular efference. J Neurosci 22:RC226.

Dickman JD, Angelaki DE (2002) Vestibular convergence patterns in vestibular nuclei neurons of alert primates. J Neurophysiol 88:3518-3533.

Draper NR, Smith H (1998) Applied regression analysis. New York: Wiley.

Ezure K, Wilson VJ (1984) Interaction of tonic neck and vestibular reflexes in the forelimb of the decerebrate cat. Exp Brain Res 54:289-292.

Fuchs AF, Robinson FR, Straube A (1993) Role of the caudal fastigial nu- cleus in saccade generation. I. Neuronal discharge pattern. J Neurophysiol 70:1723-1740.

Fukushima K, Peterson BW, Uchino Y, Coulter JD, Wilson VJ (1977) Direct fastigiospinal fibers in the cat. Brain Res 126:538-542.

Gardner EP, Fuchs AF (1975) Single-unit responses to natural vestibular stimuli and eye movements in deep cerebellar nuclei of the alert rhesus monkey. J Neurophysiol 38:627-649.

Gdowski GT, McCrea RA (1999) Integration of vestibular and head movement signals in the vestibular nuclei during whole-body rotation. J Neurophysiol 82:436-449.

Gdowski GT, McCrea RA (2000) Neck proprioceptive inputs to primate vestibular nucleus neurons. Exp Brain Res 135:511-526.

Goodwin GM, McCloskey DI, Matthews PB (1972) A systematic distortion of position sense produced by muscle fibration. J Physiol (Lond) 221:8P-9P.

Horak FB, Shupert CL, Dietz V, Horstmann G (1994) Vestibular and somatosensory contributions to responses to head and body displacements in stance. Exp Brain Res 100:93-106.

Ivanenko YP, Grasso R, Lacquaniti F (1999) Effect of gaze on postural responses to neck proprioceptive and vestibular stimulation in humans. J Physiol (Lond) 519:301-314.

Karnath HO, Sievering D, Fetter M (1994) The interactive contribution of neck muscle proprioception and vestibular stimulation to subjective straight-ahead orientation in man. Exp Brain Res 101:140-146.

Kasper J, Schor RH, Wilson VJ (1988) Response of vestibular neurons to head rotations in vertical planes. II. Response to neck stimulation and vestibular-neck interaction J Neurophysiol 60:1765-1778.

Kennedy PM, Inglis JT (2002) Interaction effects of galvanic vestibular stimulation and head position on the soleus H reflex in humans. Clin Neurophysiol 113:1709-1714.

Kyuhou S, Kawaguchi S (1987) Cerebellocerebral projection from the fastigial nucleus onto the frontal eye field and anterior ectosylvian visual area in the cat. J Comp Neurol 259:571-590.

Lackner JR, Graybiel A (1978) Some influences of touch and pressure cues on human spatial orientation. Aviat Space Environ Med 49:798-804.

Manzoni D, Pompeiano O, Srivastava UC, Stampacchia G (1983) Responses of forelimb extensors to sinusoidal stimulation of macular labyrinth and neck receptors. Archs Ital Biol 121:205-214.

Manzoni D, Pompeiano O, Andre P (1998) Convergence of directional vestibular and neck signals on cerebellar Purkinje cells. Pflügers Arch 435:617-630.

Manzoni D, Pompeiano O, Bruschini L, Andre P (1999) Neck input modifies the reference frame for coding labyrinthine signals in the cerebellar vermis: a cellular analysis. Neuroscience 93:1095-1107.

McCrea RA, Gdowski GT, Boyle R, Belton T (1999) Firing behavior of vestibular neurons during active and passive head movements: vestibulespinal and other non-eye-movement related neurons. J Neurophysiol $82: 416-428$.

Mergner T, Nardi GL, Becker W, Deecke L (1983) The role of canal-neck interaction for the perception of horizontal trunk and head rotation. Exp Brain Res 49:198-208.

Mergner T, Siebold C, Schweigart G, Becker W (1991) Human perception of horizontal head and trunk rotation in space during vestibular and neck stimulatin. Exp Brain Res 85:389-404.

Mergner T, Rottler G, Kimmig H, Becker W (1992) Role of vestibular and neck inputs for the perception of subject motion in space. Exp Brain Res 89:655-668.

Nakano K, Takimoto T, Kayahara T, Takeuchi Y, Kobayashi Y (1980) Distribution of cerebellothalamic neurons projecting to the ventral nuclei of the thalamus: an HRP study in the cat. J Comp Neurol 194:427-439.

Nashner LM, Wolfson P (1974) Influence of head position and proprioceptive cues on short latency postural reflexes evoked by galvanic stimulation of the human labyrinth. Brain Res 67:255-268.

Noda H, Sugita S, Ikeda Y (1990) Afferent and efferent connections of the oculomotor region of the fastigial nucleus in the macaque monkey. J Comp Neurol 302:330-348.

Peterson BW, Goldberg J, Bilotto G, Fuller JH (1985) Cervicocollic reflex: its dynamic properties and interaction with vestibular reflexes. J Neurophysiol 54:90-109.

Pyykko I, Aalto H, Seidel H, Starck J (1989) Hierarchy of different muscles in postural control. Acta Otolaryngol [Suppl] 468:175-180. 
Roy JE, Cullen KE (2001) Selective processing of vestibular reafference during self-generated head motion. J Neurosci 21:2131-2142.

Sasaki K, Kawaguchi S, Oka H, Sakai M, Mizuno N (1976) Electrophysiological studies on the cerebellocerebral projections in monkeys. Exp Brain Res 24:495-507.

Siebold C, Glonti L, Glasauer S, Buttner U (1997) Rostral fastigial nucleus activity in the alert monkey during three dimensional passive head movements. J Neurophysiol 77:1432-1446.

Siebold C, Kleine JF, Glonti L, Tchelidze T, Buttner U (1999) Fastigial nucleus activity during different frequencies and orientations of vertical vestibular stimulation in the monkey. J Neurophysiol 82:34-41.

von Holst E, Mittelstaedt H (1950) Das reafferenzprinzip. Naturwissenschaften 37:464-476.
Voogd J (1989) Parasagittal zones and compartments of the anterior vermis of the cat cerebellum. Exp Brain Res 17:3-19.

Wilson VJ, Uchino Y, Susswein A, Fukushima K (1977) Properties of direct fastigiospinal fibers in the cat. Brain Res 126:543-546.

Wilson VJ, Schor RH, Suzuki I, Park BR (1986) Spatial organization of neck and vestibular reflexes acting on the forelimbs of the decerebrate cat. J Neurophysiol 55:514-526.

Wilson VJ, Yamagata Y, Yates BJ, Schor RH, Nonaka S (1990) Response of vestibular neurons to head rotations in vertical planes. III. Response of vestibulocollic neurons to vestibular and neck stimulation. J Neurophysiol 64:1695-1703.

Zhou W, Tang BF, King WM (2001) Responses of rostral fastigial neurons to linear acceleration in an alert monkey. Exp Brain Res 139:111-115. 